Revista de Matemática: Teoría y Aplicaciones 2012 19(2) : 183-199

CIMPA - UCR ISSN: 1409-2433

\title{
NUEVAS MEDIDAS DE COMPACIDAD GEOMÉTRICA PARA EL DISEÑO DE ZONAS
}

\section{NEW GEOMETRICAL COMPACTNESS MEASURES FOR ZONES DESIGN}

\author{
ERIC A. RINCÓN-GARCÍA* \\ Miguel Ángel Gutiérrez-Andrade ${ }^{\dagger}$ \\ Sergio G. DE-LOS-Cobos-Silva \\ PEDRO LARA-VELÁZQUEZ ${ }^{\S}$
}

Received: 16 Dec 2009; Revised: 10 Nov 2011; Accepted: 10 May 2012

\footnotetext{
*Universidad Autónoma Metropolitana-Azcapotzalco, Departamento de Sistemas, Av. San Pablo 180 Col. Reynosa Tamaulipas C.P. 02200, México D.F., México. EMail: caracol_loco@yahoo.com

${ }_{\dagger}^{\dagger}$ Universidad Autónoma Metropolitana-Iztapalapa, Departamento de Ingeniería Eléctrica, Av. Michoacán y La Purísima s/n, Col. Vicentina, Del. Iztapalapa, México D.F. C.P. 09340, México. E-Mail: gamma@xanum.uam.mx

${ }^{\ddagger}$ Misma dirección que/Same address as: M.A. Gutiérrez. E-Mail: cobos@xanum.uam.mx

${ }^{\S}$ Misma dirección que/Same address as: E.A. Rincón. E-Mail: pedro_lara@correo.azc.uam.mx
} 


\title{
Resumen
}

El diseño de zonas compactas es un problema que ha sido estudiado por su influencia en la creación de zonas con formas regulares, las cuales resultan más fáciles de análizar, estudiar o administrar. En este trabajo se propone un nuevo método para medir la compacidad, mediante la transformación de los espacios geográficos originales, en figuras formadas con celdas cuadradas, las cuales son utilizadas para cuantificar la similitud entre la zona original y una zona ideal con contornos rectos. Este procedimiento fue aplicado para diseñar zonas electorales compactas, conexas y con equilibrio poblacional, en una configuración topográfica que favorece la creación de formas retorcidas y difusas. Los resultados obtenidos, muestran que el nuevo método favorece la creación de zonas con formas rectas, sin afectar, de manera importante el equilibrio poblacional, dando como resultado zonas de buena calidad tanto por su forma como por el número de habitantes que contiene.

Palabras clave: Distritación, compacidad, recocido simulado, SIG.

\begin{abstract}
The design of compact zones has been studied because of its influence in the creation of zones with regular forms, which are easier to analyze, to investigate or to administer. This paper propose a new method to measure compactness, by means of the transformation of the original geographical spaces, into figures formed with square cells, which are used to measure the similarity between the original zone and an ideal zone with straight forms. The proposed method was applied to design electoral zones, which must satisfy constraints of compactness, contiguity and population balance, in a topographical configuration that favors the creation of twisted and diffuse shapes. The results show that the new method favors the creation of zones with straight forms, without an important effect to the population balance, which are considered zones of high quality.
\end{abstract}

Keywords: Redistricting, compactness, simulated annealing, GIS.

Mathematics Subject Classification: 90C59, 90C29, 68T20.

\section{Introducción}

El diseño de zonas consiste en agrupar pequeños espacios o unidades geográficas básicas (UGB) para formar zonas que satisfacen las restricciones y optimizan los objetivos impuestos por el problema. Uno de los objetivos más comunes en este tipo de problemas es la generación de zonas compactas, caracterizadas por tener formas regulares y homogéneas dentro de un espacio limitado. Esta propiedad resulta muy valiosa porque 
dan claridad en la delimitación de las zonas para fines de administración [3], mantenimiento y control [9], para disminuir el costo de construcción de servicios tanto públicos como privados [12], o para disminuir tiempos de traslado o recorrido dentro de la zona [11]. Sin embargo, en las aplicaciones reales, la compacidad es sólo uno de los objetivos buscados y, normalmente, diferentes objetivos en un mismo problema competirán entre sí, de tal forma que la mejora de uno de ellos puede implicar el deterioro de otro. Por lo cual, resulta importante establecer un método que garantice la obtención de zonas con formas regulares sin afectar, de manera significativa, a los otros objetivos del problema.

En este trabajo se propone un método para medir la compacidad de las zonas mediante la transformación de los espacios geográficos originales en figuras construidas con celdas cuadradas, y el uso de dos medidas de compacidad, que utilizan las nuevas figuras, para promover la creación de zonas con formas rectas y penalizar las figuras retorcidas o dispersas. Estas propuestas fueron aplicadas en el estado de Baja California Norte, México, para diseñar zonas electorales compactas, conexas y con equilibrio poblacional. Los resultados obtenidos muestran que el método y las nuevas medidas propuestas, no solo cumplen con el objetivo de generar zonas compactas, sino que además, evitan la interferencia con el equilibrio poblacional, dando como resultado zonas de muy buena calidad, tanto por su forma como por el número de habitantes que contienen.

\section{Complejidad}

Una de las razones por las que el diseño de zonas es un problema difícil, se debe al tamaño del espacio de soluciones, que en los problemas reales, generalmente hace irrealizable cualquier tentativa de enumerar explícitamente todas las soluciones factibles. Aún para un pequeño número de unidades geográficas y zonas, la cantidad de posibles arreglos es enorme, por ejemplo, el número total de soluciones para dividir $n$ UGB en $k$ zonas está dado por el número de Stirling del segundo tipo ([1]).

En casos especiales, se puede reducir el tamaño del espacio solución, por ejemplo, si se generan zonas con exactamente $r$ UGB, el número de soluciones está dado por:

$$
S^{\prime}(n, k, r)=\frac{n !}{(r !)^{k}}
$$

Para ejemplificar el crecimiento del espacio de soluciones en este caso, se muestra, en la Tabla 1, el número posible de planes de zonificación que podrían usarse para dividir un estado hipotético en dos zonas con la 
única restricción de que cada zona contenga la misma cantidad de unidades geográficas.

\begin{tabular}{l|cccccc}
\hline \hline & \multicolumn{5}{|c}{ Unidades Geográficas Básicas } \\
\hline $\begin{array}{l}\text { Total de } \\
\text { unidades }\end{array}$ & 10 & 20 & 50 & 100 & 150 & 250 \\
$\begin{array}{l}\text { Unidades } \\
\text { por zona }\end{array}$ & 5 & 10 & 25 & 50 & 75 & 125 \\
$\begin{array}{l}\text { Número } \\
\text { de planes }\end{array}$ & 945 & $6.5 \times 10^{8}$ & $5.8 \times 10^{31}$ & $2.73 \times 10^{78}$ & $6.09 \times 10^{78}$ & $4 \times 10^{245}$ \\
\hline \hline
\end{tabular}

Tabla 1: Planes de zonificación con el mismo número de unidades.

O bien, si se considera que las UGB están conectadas en una cadena y cada una es contigua solamente a dos vecinos, exceptuando a las ubicadas en los extremos, el número de soluciones está dado por [2]:

$$
S^{\prime \prime}(n, k)=\frac{(n-1)}{(k-1) !(n-k) !} .
$$

Para ejemplificar el crecimiento del espacio de soluciones en este caso, en la Tabla 2 se pueden observar el número posible de planes de zonificación que podrían usarse para dividir un estado hipotético en cuatro u ocho zonas.

\begin{tabular}{l|cccccc}
\hline \hline & \multicolumn{6}{|c}{ Unidades Geográficas Básicas } \\
\hline $\begin{array}{l}\text { Total de } \\
\text { unidades }\end{array}$ & 50 & 50 & 100 & 100 & 150 & 150 \\
$\begin{array}{l}\text { Número } \\
\text { de zonas }\end{array}$ & 4 & 8 & 4 & 8 & 4 & 8 \\
$\begin{array}{l}\text { Número } \\
\text { de planes }\end{array}$ & 18424 & $8.6 \times 10^{7}$ & 156849 & $1.49 \times 10^{10}$ & 540274 & $2.8 \times 10^{11}$ \\
\hline \hline
\end{tabular}

Tabla 2: Planes de zonificación con unidades en una cadena.

De esta forma, se destaca que incluso en casos muy especiales, el espacio de soluciones crece rápidamente. Aunado a lo anterior, se ha demostrado que el diseño de zonas compactas en términos de complejidad computacional es un problema NP-duro ([1], [5]).

Por lo anterior, el diseño de zonas conexas con equilibrio poblacional y compacidad, como ocurre en zonas electorales, será un problema al menos NP-duro, y por lo tanto, tratar de encontrar la solución óptima puede implicar tiempos de búsqueda demasiado largos. 


\section{Compacidad en celdas cuadradas}

Para utilizar la compacidad en celdas, la figura estudiada debe ser transformada en un conjunto de celdas cuadradas del mismo tamaño. En la figura 1 se observa el Distrito Federal de México, formado con un conjunto de celdas del mismo tamaño.

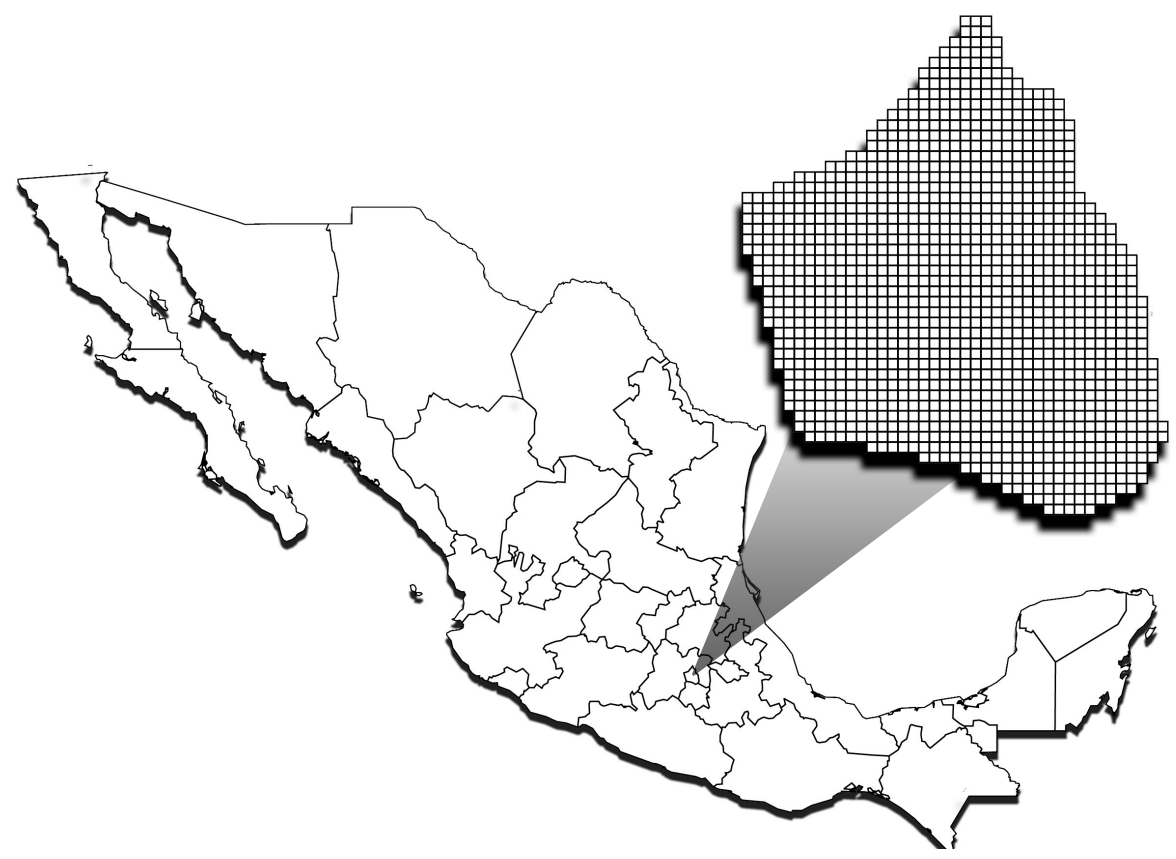

Figura 1: Distrito Federal formado con celdas cuadradas.

Una vez que se obtiene la nueva figura, se puede observar que cada celda y sus lados sólo tienen dos opciones, formar parte del contorno o estar en el interior. A partir de lo anterior, se presentan las siguientes definiciones:

Definición 1 El área en celdas es el número de celdas que forman la figura.

Definición 2 El perímetro en celdas es el número de celdas que forman el contorno de la figura.

Definición 3 El perímetro es el número de lados que se encuentran en el contorno de la figura.

Se observa que una zona con forma retorcida, alargada y dispersa tendrá un área en celdas menor que el mínimo rectángulo formado por 
celdas cuadradas que la inscribe. Así mismo, su perímetro y su perímetro en celdas será mayor que el del mínimo rectángulo, formado por celdas cuadradas, ver figura 2 .

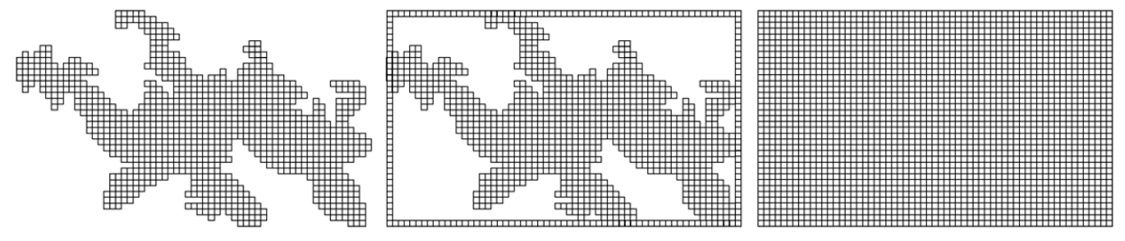

Figura 2: Zona analizada y mínimo rectángulo que la inscribe.

Por lo tanto, la máxima compacidad se obtendrá cuando la zona y el rectángulo que la inscribe tengan, aproximadamente, la misma área, perímetro y perímetro en celdas. Con lo cual, se favorece el diseño de zonas con pocas irregularidades y formas rectas.

Para medir la diferencia del área en celdas, se aplicará la siguiente operación:

$$
\frac{A C_{R}}{A C_{Z}}
$$

donde: $A C_{Z}$, es el área en celdas de la zona y $A C_{R}$, es el área en celdas del mínimo rectángulo que inscribe a la zona.

Con este planteamiento, todas las zonas tendrán valores mayores que 1 y las figuras más compactas tendrán valores más pequeños.

Para medir la diferencia del perímetro en celdas se aplicará la siguiente operación:

$$
\frac{P C_{Z}}{P C_{R}}+\frac{P C_{R}}{P C_{Z}}
$$

donde: $P C_{Z}$, es el perímetro en celdas de la zona, $P C_{R}$, es el perímetro en celdas del mínimo rectángulo que inscribe a la zona.

Se observa que esta ecuación es mayor que 2 para todas las zonas por lo cual se realiza la siguiente modificación:

$$
\frac{P C_{Z}}{P C_{R}}+\frac{P C_{R}}{P C_{Z}}-2
$$

Para medir la diferencia del perímetro se aplicará la siguiente operación:

$$
\frac{P_{Z}}{P_{R}}
$$

donde: $P_{Z}$, es el perímetro en celdas de la zona, $P_{R}$, es el perímetro en celdas del mínimo rectángulo que inscribe a la zona. 
Debe observarse que los valores de esta ecuación siempre son mayores que 1, por lo cual se hace la siguiente modificación:

$$
\frac{P_{Z}}{P_{R}}-1
$$

Con estas ecuaciones, se establecen dos medidas diferentes para determinar la compacidad de las zonas estudiadas.

La primera medida compara el área en celdas y el perímetro en celdas de la zona estudiada y del mínimo rectángulo que la inscribe, de la siguiente manera:

$$
\text { Compacidad } 1=\left(\frac{A C_{R}}{A C_{Z}}\right)\left(\frac{P_{Z}}{P_{R}}-1\right) .
$$

La segunda medida compara el perímetro y el perímetro en celdas de la zona estudiada y del mínimo rectángulo que la inscribe, de la siguiente manera:

$$
\text { Compacidad } 2=\frac{P C_{Z}}{P C_{R}}+\frac{P C_{R}}{P C_{Z}}+\frac{P_{Z}}{P_{R}}-3 .
$$

De esta forma, ambas medidas le asignan valores muy cercanos a cero a las zonas más compactas.

Es importante destacar que ambas propuestas requieren de pocos pasos para ser calculadas, lo cual favorecerá el desempeño de cualquier algoritmo que necesite calcular en varias ocasiones el costo de las zonas diseñadas.

\section{Optimización de zonas electorales}

Como se mencionó anteriormente, el diseño de zonas aparece en diversas aplicaciones. Sin embargo, el caso más conocido por su influencia en los resultados de procesos electorales, es el diseño de zonas electorales o distritación política. En este tipo de aplicaciones, se deben agrupar UGB, generalmente unidades administrativas, en un número predeterminado de zonas o distritos de tal forma que se garantice la democracia del proceso político. Para lograrlo, se imponen condiciones que varían dependiendo del país o incluso del estado en el que se realiza la distritación. No obstante, existen tres criterios que se consideran imprescindibles en todo ejercicio democrático [4], [6], [10]:

- Zonas contiguas (conexidad), para facilitar la administración durante y después del proceso electoral, así como para evitar manipulación electoral.

- Zonas con el mismo número de electores (equilibrio poblacional), para garantizar la igualdad de representación política. 
- Zonas sin formas retorcidas, alargadas y dispersas (compacidad), para evitar la manipulación conocida como gerrymandering, en la cual se diseñan zonas conexas y con equilibrio poblacional pero que favorecen a un sector político o social.

En este trabajo, se define una función objetivo que minimiza el costo del equilibrio poblacional y la compacidad, mientras que la conexidad es garantizada por el algoritmo de búsqueda.

El equilibrio poblacional será medido con la fórmula usada por el Instituto Federal Electoral de México (IFE) en la distritación para las elecciones del 2006, la cual establece un intervalo máximo permisible para poder realizar la distritación dada por:

$$
\frac{P_{T}}{n}-d_{a} \leq P_{D} \leq \frac{P_{T}}{n}+d_{a},
$$

donde: $P_{D}$ es la población del distrito, $P_{T}$ es la población total de la entidad, $d_{a}$, es la desviación poblacional máxima aceptable para la entidad, y $n$ es el número de distritos electorales que se deben generar en la entidad.

Reagrupando términos se obtiene que:

$$
-1 \leq\left(\frac{P_{T}}{d_{a}}\right)\left(\frac{P_{D}}{P_{T}}-\frac{1}{n}\right) \leq+1 .
$$

Finalmente, el costo poblacional de cada distrito $D$, es calculado por:

$$
\left(\frac{P_{T}}{d_{a}}\right)^{2}\left(\frac{P_{D}}{P_{T}}-\frac{1}{n}\right)^{2}
$$

De esta forma, mientras menor sea el costo de una solución, mayor será su equilibrio poblacional.

Para determinar la compacidad de las zonas se utilizarán por separado las medidas propuestas en este trabajo.

$$
\text { Compacidad } 1=\left(\frac{A C_{R}}{A C_{Z}}\right)\left(\frac{P_{Z}}{P_{R}}-1\right) .
$$

O bien,

$$
\text { Compacidad } 2=\frac{P C_{Z}}{P C_{R}}+\frac{P C_{R}}{P C_{Z}}+\frac{P_{Z}}{P_{R}}-3 .
$$

También en este caso, las zonas más compactas tendrán valores más pequeños.

Antes de presentar la función objetivo se agrega la siguiente notación: $f_{1}(P)$, costo del equilibrio poblacional asociado al plan de zonificación $P$, $f_{2}(P)$, costo de compacidad asociado al plan de zonificación $P, \alpha_{1}, \alpha_{2}$ son factores de ponderación. 
Utilizando esta notación se plantea la función objetivo de la siguiente manera:

$$
\text { Minimizar } f(P)=\alpha_{1} f_{1}(P)+\alpha_{2} f_{2}(P) \text {. }
$$

Con este planteamiento, la función objetivo buscará que el resultado óptimo tenga el mejor equilibrio poblacional y la mayor compacidad posible.

\section{$5 \quad$ Recocido simulado}

Como se mencionó anteriormente, el tiempo necesario para encontrar el óptimo en este tipo de problemas, podría ser excesivo debido al tamaño del espacio solución. Por este motivo, es necesario diseñar un método capaz de generar soluciones de buena calidad en tiempo de cómputo aceptable. Esto implicará que no se podrán revisar todas las soluciones factibles y por lo tanto, no se podrá garantizar la optimalidad. Los métodos de búsqueda con estas características son llamados heurísticos y varios de ellos han sido aplicados al diseño de zonas.

En este trabajo, se diseñó un algoritmo basado en recocido simulado por tratarse de una heurística cuya simplicidad y buenos resultados, en una amplia variedad de problemas NP-Duros, lo han convertido en una herramienta muy popular, con aplicaciones en diferentes áreas de optimización.

El concepto fue introducido en el campo de la optimización combinatoria a inicios de la década de los 80 por Kirkpatrick [7]. Su fundamentación se basa en el trabajo de Metropolis en el campo de la termodinámica estadística [8]. En dicho trabajo, se modela el proceso de recocido de sólidos simulando los cambios energéticos en un sistema de partículas conforme decrece la temperatura, hasta converger a un estado estable (congelado).

Esta heurística comienza con una solución y un parámetro de control $T_{0}$ llamado temperatura. En cada iteración se genera de manera aleatoria una solución vecina, $P_{B}$, si la nueva solución mejora el valor de la función objetivo con respecto a la solución actual, $P_{A}, f\left(P_{A}\right)-f\left(P_{B}\right)>0$, ésta última es reemplazada. Cuando la nueva solución no mejora el valor de la función objetivo, se puede aceptar el cambio de la solución actual con cierta probabilidad dada por:

$$
\exp \left(\frac{f\left(P_{A}\right)-f\left(P_{B}\right)}{T}\right)
$$

donde $f\left(P_{A}\right)$ es el costo de la solución actual, $f\left(P_{B}\right)$ es el costo de la solución vecina, y $T$ es la temperatura del proceso. 
Se recomienda que al inicio del algoritmo la temperatura sea suficientemente alta para permitir todo, o casi todo, movimiento, es decir, que la probabilidad de pasar de la solución $P_{A}$ a la solución $P_{B}$ sea muy alta, sin importar la diferencia entre los costos de ambas soluciones, $f\left(P_{A}\right)-f\left(P_{B}\right)$.

Conforme el algoritmo avanza, el valor de la temperatura se disminuye mediante un coeficiente de enfriamiento, $0<\alpha<1$, pero cada valor es mantenido estable durante $L$ iteraciones, para permitir que el algoritmo explore distintas soluciones con la misma probabilidad de aceptación. Debe observarse que al inicio, cuando la temperatura es alta, se tiene una mayor probabilidad de aceptar soluciones inferiores, lo cual permite la exploración del espacio solución y evita la convergencia prematura a mínimos locales. Sin embargo, conforme disminuye el valor de la temperatura, el algoritmo se hace más selectivo y difícilmente acepta soluciones inferiores, iniciando una búsqueda que lo guía hacia un mínimo local. Finalmente, el algoritmo es detenido cuando la temperatura alcanza un valor límite, $T_{f}$, y devuelve la mejor solución encontrada.

En este trabajo, los parámetros de temperatura inicial $T_{0}$, coeficiente de enfriamiento $\alpha$, temperatura final $T_{f}$ y el número de soluciones $L$ visitadas en cada temperatura, se mantendrán fijos durante todo el proceso, y para determinar los valores adecuados se tomarán las siguientes consideraciones:

a) Temperatura inicial $T_{0}$. La temperatura inicial $T_{0}$ debe permitir casi todo movimiento, por lo cual será lo suficientemente alta para que el algoritmo acepte al menos el $80 \%$ de las soluciones inferiores encontradas.

b) Temperatura final $T_{f}$. La temperatura final indicará el momento en el cual el algorítmo debe detenerse, por lo tanto, debe ser suficientemente pequeña para aceptar menos del $1 \%$ de las soluciones inferiores encontradas.

c) Factor de enfriamiento $\alpha$. Un método muy usado para disminuir la temperatura es dado por la función: $T_{k+1}=\alpha T_{k} \quad k=0,1, \ldots$ donde $\alpha$ es una constante positiva menor que 1 , normalmente entre 0.8 y 0.99 .

d) Criterio de cambio de la temperatura $L$. La cantidad de iteraciones que se hacen en cada temperatura, afecta tanto el tiempo de ejecución del algoritmo como su capacidad para explorar el espacio solución y por lo tanto, las posibilidades de encontrar soluciones de buena calidad. Para lograr un equilibrio adecuado, se recomienda establecer un valor fijo relacionado con el número de vecindades del problema. 
Otro factor importante para el desempeño del algoritmo, es la forma en que se definen a las soluciones vecinas o vecindario de la solución actual. En este trabajo, las soluciones vecinas serán generadas de la siguiente manera. De la solución actual se elige de manera aleatoria una UGB que pueda ser cambiada de zona, de tal forma que el resultado sea otra solución factible. Primero se garantiza la conexidad de las nuevas zonas, mediante la elección de una UGB que se encuentre en la colindancia entre dos zonas y que, al ser cambiada, no provoque una disconexión ni en la zona a la cual pertenece actualmente, ni en la zona a la cual pertenecerá. Después, se revisa que el cambio no disminuya el número de zonas. De esta forma, cada solución vecina es una solución factible que se diferencia de la anterior sólo por la ubicación de una UGB.

Por último, se explicará la forma en que es construida la solución inicial. Sin embargo, es importante mencionar que recocido simulado se caracteriza por su capacidad para obtener soluciones de buena calidad, sin importar la solución inicial que se utilice. El algoritmo empieza con la construcción de una solución factible, para lo cual se seleccionan de manera aleatoria $r$ UGB y las marca como zonas iniciales. Después, a cada zona le anexa una UGB vecina que aún no forme parte de ninguna zona y continúa hasta incluir todas las UGB. De esta forma se obtiene una solución factible con $r$ zonas conexas ajenas que incluyen a todas las UGB.

\section{Aplicación}

Para determinar la eficiencia de la compacidad en celdas, se aplicaron las medidas propuestas al estado mexicano de Baja California Norte, ver figura 3. En este estado viven 2,487,367 electores, y se encuentra formado por 319 UGB que deben ser divididas en 8 zonas electorales, con un porcentaje de desviación poblacional máximo de $d_{a}=15 \%$ de acuerdo con lo estipulado por el IFE. Por último, se considerará una población nacional de 97,483,412 electores, según el censo practicado por en el año 2000.

En la figura 4 se presentan las 319 UGB que forman el estado de Baja California Norte y se puede observar su gran variedad en formas y tamaños, lo cual se convierte en una de las principales complicaciones al tratar de diseñar zonas compactas.

Para aplicar la compacidad por celdas, el estado fue dividido en celdas cuadradas de 10 metros por lado. Para hacerlo, se utilizó el sistema de información geográfica Arcview 3.0, que permite obtener, a partir de los archivos originales en formato shape, archivos en formato grid formados con celdas cuadradas del tamaño deseado. De esta forma, cada unidad geográfica es transformada en un conjunto de celdas cuadradas. Para conocer 


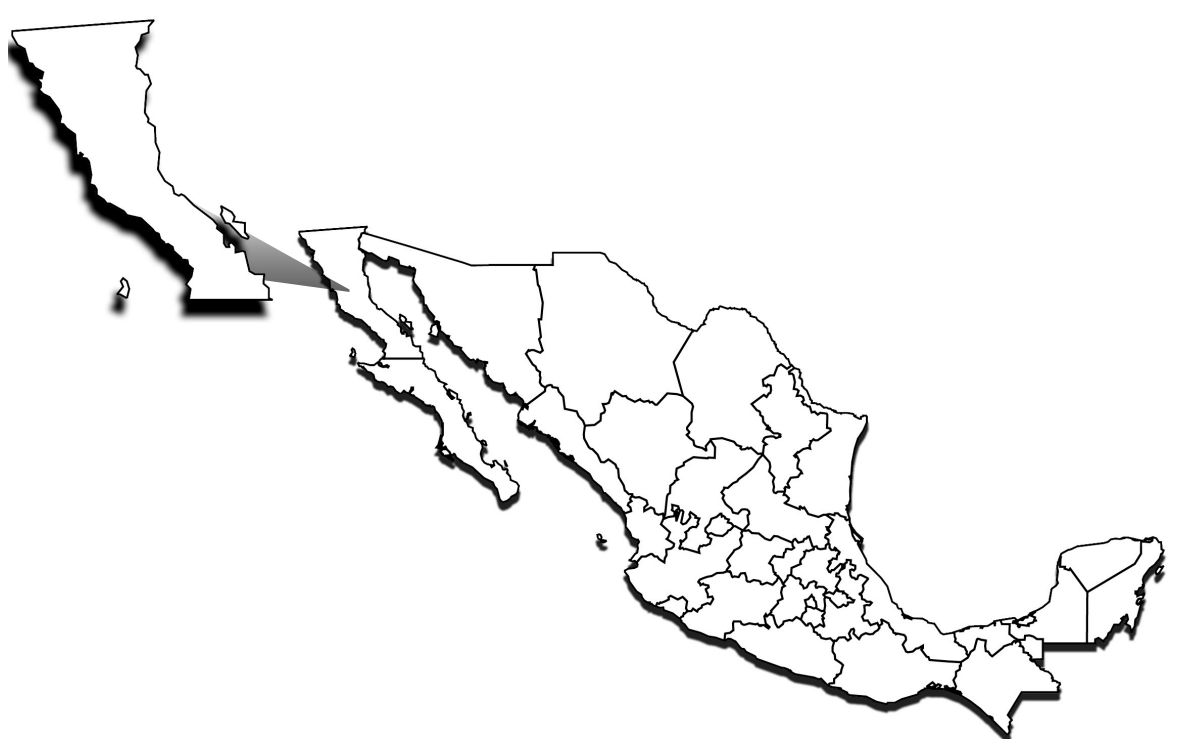

Figura 3: Zona analizada y mínimo rectángulo que la inscribe.

el número de celdas y lados que forman a cada UGB y al perímetro que comparten con las unidades vecinas, se diseñó un scrip en Avenue, lenguaje de programación en Arcview, que lee los datos del archivo grid y los envía a archivos de texto, que son utilizados posteriormente por el heurístico diseñado en este trabajo.

Por último, se diseñó un algoritmo basado en recocido simulado, escrito en $\mathrm{C}^{++}$, para realizar la búsqueda en el espacio de soluciones factibles del problema estudiado. En cada iteración, el algoritmo de optimización calcula el número de habitantes, el perímetro y el área en celdas, o el perímetro en celdas, de cada zona dependiendo de la medida de compacidad que se este utilizando. Establece el costo de cada solución, y determina si las nuevas soluciones son aceptadas o rechazadas según los criterios de recocido simulado.

Para determinar los valores de los factores del programa de enfriamiento, se realizaron varias corridas que permitieron conocer el comportamiento de las soluciones visitadas por el algoritmo. Para ambas medidas de compacidad, se establecieron los siguientes valores:

- Temperatura inicial $T_{0}=10$, con una aceptación superior al $80 \%$.

- Temperatura final $T_{f}=0.005$, con una aceptación menor al 1\%.

- Iteraciones para cambio de la temperatura $L=1000$.

- Factor de enfriamiento $\alpha=0.97$. 


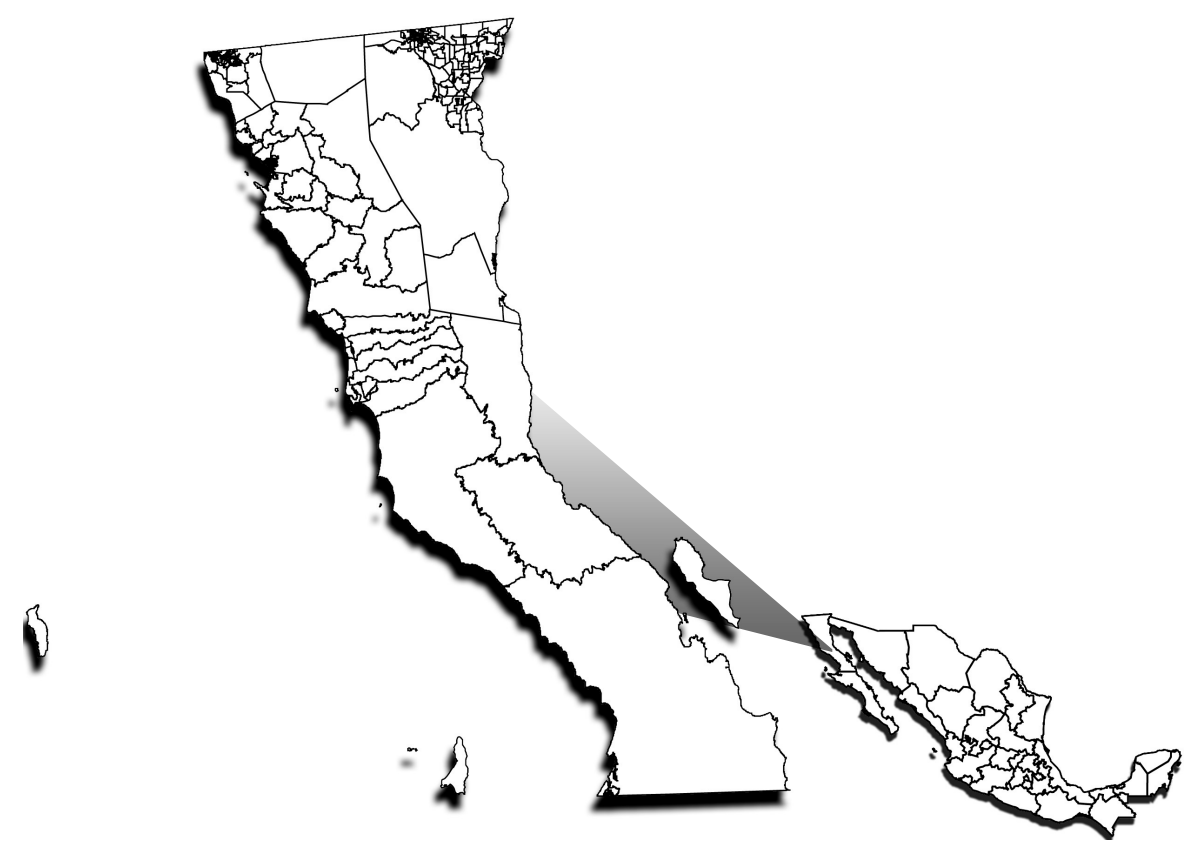

Figura 4: Unidades geográficas básicas de Baja California Norte.

Para determinar los valores de los factores de ponderación también se realizaron varias corridas, y se observó cómo variaban los costos de equilibrio poblacional y compacidad tanto en las soluciones visitadas como en las soluciones finales. Se notó que se favorecían las soluciones con un buen equilibrio poblacional, dando como resultado valores relativamente altos en la compacidad. Para equilibrar está situación, en ambas medidas se asignó un valor de ponderación bajo para el equilibrio poblacional en comparación con el valor asignado a la compacidad.

- En la medida 1 se utilizaron $\alpha_{1}=0.2$ y $\alpha_{2}=0.8$.

- En la medida 2 se utilizaron $\alpha_{1}=0.1$ y $\alpha_{2}=5$.

Es importante destacar que la diferencia tan amplia en los factores de ponderación no implica ninguna ventaja de la compacidad sobre el equilibrio poblacional, de hecho, los resultados obtenidos muestran un equilibrio poblacional muy bueno en las zonas generadas con ambas medidas.

En la Tabla 3 se presentan los costos del equilibrio poblacional de todas las zonas propuestas como solución final al aplicar la medida de compacidad 1. Se observa que las más penalizadas son las que tienen un mayor porcentaje de desviación con respecto a la población ideal, de aproximadamente 310,921 electores. De hecho, la máxima desviación poblacional es 


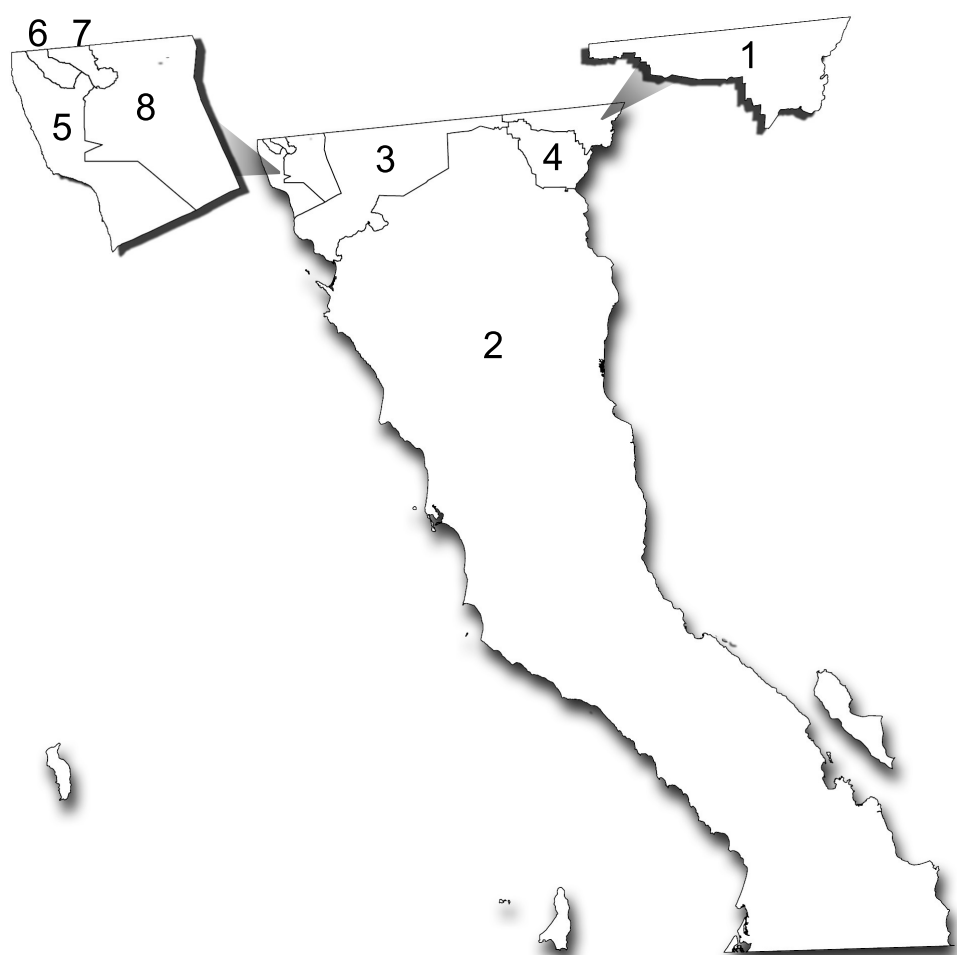

Figura 5: Zonas generadas al aplicar la medida de compacidad 1.

del $7.65 \%$ en la zona 6 , casi la mitad de la desviación máxima aceptada del $15 \%$.

Para la compacidad, se obtiene un costo mayor a 1 en la zona 2. Sin embargo, las zonas son congruentes con los objetivos propuestos, al favorecer formas rectas y evitar figuras retorcidas, alargadas y dispersas, ver figura 4.

En la Tabla 4 se presentan los costos del equilibrio poblacional de todas las zonas propuestas como solución final al aplicar la medida de compacidad 2. En este caso, la máxima desviación poblacional es del $5.48 \%$ en la zona 8, menos de la mitad de la desviación máxima aceptada del 15\%.

Para la compacidad, se obtienen costos menores que 1 en todas las zonas. Dando como resultado zonas de buena calidad tanto por su forma como por el número de electores que contienen, ver figura 6 .

\section{Conclusiones}

En este trabajo se proponen dos formas diferentes para medir la compacidad mediante el uso de celdas cuadradas, que no habían sido utilizadas 


\begin{tabular}{c|cccc}
\hline \hline Zona & Electores & $\begin{array}{c}\text { Equilibrio } \\
\text { poblacional }\end{array}$ & $\begin{array}{c}\text { Porcentaje de } \\
\text { desviación (\%) }\end{array}$ & Compacidad \\
\hline 1 & 310617 & 0.000008 & 0.098 & 0.241573 \\
2 & 301455 & 0.007543 & 3.04 & 2.1222714 \\
3 & 2299877 & 0.010268 & 3.55 & 0.464406 \\
4 & 300950 & 0.008369 & 3.21 & 0.432751 \\
5 & 315749 & 0.001962 & 1.55 & 0.413522 \\
6 & 334711 & 0.047646 & 7.65 & 0.051064 \\
7 & 323426 & 0.013165 & 4.02 & 0.233737 \\
8 & 300582 & 0.008999 & 3.32 & 0.264918 \\
\hline \hline
\end{tabular}

Tabla 3: Costos de compacidad por zona al aplicar la medida 1.

\begin{tabular}{c|cccc}
\hline \hline Zona & Electores & $\begin{array}{c}\text { Equilibrio } \\
\text { poblacional }\end{array}$ & $\begin{array}{c}\text { Porcentaje de } \\
\text { desviación }(\%)\end{array}$ & Compacidad \\
\hline 1 & 305839 & 0.00108704 & 1.63 & 0.076195 \\
2 & 307870 & 0.00039178 & 0.98 & 0.895008 \\
3 & 303124 & 0.00255882 & 2.51 & 0.179475 \\
4 & 296066 & 0.00928831 & 4.78 & 0.169553 \\
5 & 322497 & 0.00564060 & 3.72 & 0.107834 \\
6 & 304354 & 0.00181516 & 2.11 & 0.099976 \\
7 & 319654 & 0.00321024 & 2.81 & 0.166659 \\
8 & 327963 & 0.01222492 & 5.48 & 0.126432 \\
\hline \hline
\end{tabular}

Tabla 4: Costos de compacidad por zona al aplicar la medida 2.

en el diseño de zonas, al menos hasta donde se tiene conocimiento. El método propuesto, permite que ambas medidas puedan ser utilizadas en cualquier tipo de problema geográfico. Más aún, el uso de un sistema de información geográfica facilita la creación de celdas tan grandes o pequeñas como el problema lo requiera.

Para explorar el espacio de soluciones se diseñó un algoritmo basado en recocido simulado, por ser una heurística ampliamente reconocida debido a su eficiencia en la resolución de problemas complejos y a su capacidad para obtener soluciones finales que no dependen de la solución inicial, lo cual es una condición muy importante tanto en procesos electorales que buscan garantizar la democracia, como en problemas que requieren diversidad en soluciones de buena calidad.

Tanto el modelo como la nueva medida fueron probados en el estado de Baja California Norte, México, cuya configuración topográfica complica el diseño de zonas compactas. Las soluciones obtenidas por el algoritmo 


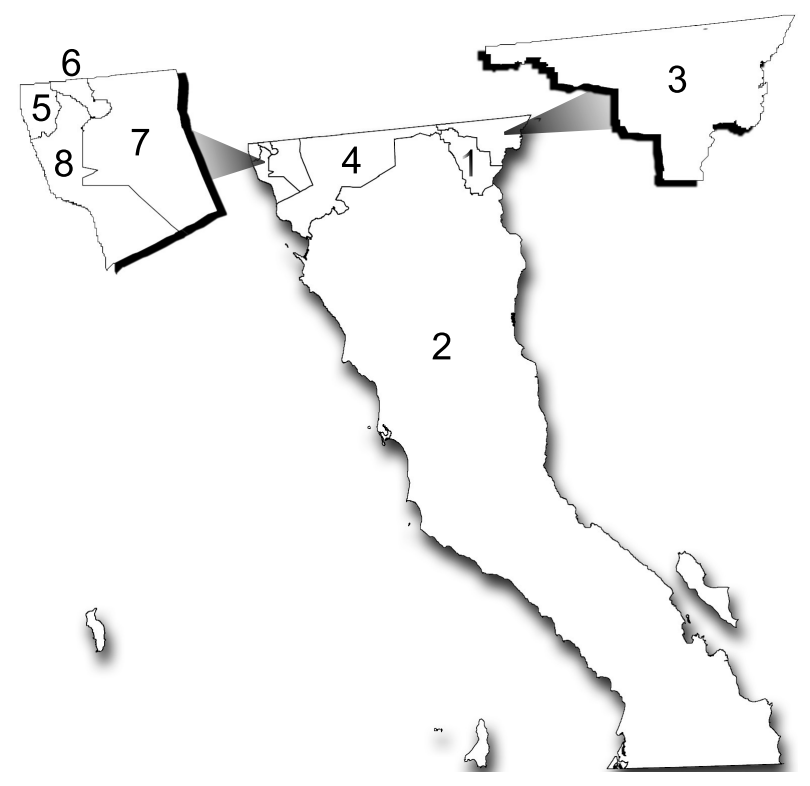

Figura 6: Zonas generadas al aplicar la medida de compacidad 2.

heurístico probaron que las medidas propuestas diseñan zonas compactas al tiempo que mantienen el equilibrio poblacional dentro de los límites establecidos. De hecho, todas las zonas diseñadas por la medida de compacidad 1, tienen un porcentaje de desviación poblacional menor que la mitad del límite permitido. De esta forma, se comprobó que ambas medidas son capaces de generar zonas de alta calidad, tanto por su forma como por el número de electores que contiene, dando como resultado planes de zonificación electoral que difícilmente serían rechazados.

Por último, las operaciones propuestas para medir la compacidad en celdas, son sencillas y requieren de muy poco tiempo para ser ejecutadas, lo cual se convierte en una cualidad muy valiosa, ya que, calcular el costo de cada solución visitada puede afectar considerablemente el tiempo de ejecución de cualquier algoritmo. Más aún, si ya se cuenta con un algoritmo o con otras medidas de compacidad para el diseño de zonas, se puede añadir la compacidad en celdas y la eficiencia del proceso de búsqueda no se verá afectada.

\section{Referencias}

[1] Altman, M. (1997) "Is automation the answer: the computational complexity of automated redistricting", Rutgers Computer and Law Technology Journal, 23(1): 81-141. 
[2] Baçao, F.; Lobo, V.; Painho, M. (2005) "Applying genetic algorithms to zone design", Soft Comput. 9: 341-348.

[3] Bergey, P.; Ragsdale, C.; Hoskote M. (2003) "A decision support system for the electrical power districting problem", Decision Support Systems 36: 1-17.

[4] Chou, C.; Li, S.P. (2007) "Spin systems and political districting problem", Journal of Magnetism and Magnetic Materials 310: 2889-2891.

[5] Gilbert, K.C.; Holmes, D.D.; Rosenthal, R.E. (1985) "A multiobjective discrete optimization model for land allocation", Management Science 31(12): 1509-1522.

[6] Gutiérrez-Andrade, M.A.; Rincón-García, E.A. (2009) "Redistriccting by square cells", Lectures Notes in Artificial Intelligence 5845, Springer, New York: 669-679.

[7] Kirkpatrick, S.; Gellat, C.D.; Vecchi, M.P. (1983) "Optimization by simulated annealing", Science 220: 671-680.

[8] Metropolis, N.; Rosenbluth, A.W.; Rosenbluth, M.N.; Teller, A.H.; Teller, E. (1953) "Equation of state calculation by fast computing machines", Journal of Chemistry Physics 21: 1087-1091.

[9] Muyldermans, L.; Cattrysse, D.; Van Oudheusden, D.; Lotan, T. (2002) "Districting for salt spreading operations", European Journal of Operational Research 139: 521-532.

[10] Ricca, F.; Simeone, B. (2008) "Local search algorithms for political districting", European Journal of Operational Research 189: 14091426 .

[11] Ríos-Mercado, R.Z.; Fernández, E. (2009) "A reactive GRASP for comercial territory design problem with multiple balancing requirements", Computers \& Operations Research 36: 755-776.

[12] Williams, J.C. (2003) "Convex land acquisition with zero-one programming", Environment and Planning B: Planning and Design 30: 255-270. 
